EPJ Web of Conferences 111, 05003 (2016)

DOI: $10.1051 /$ epjconf/201611105003

(C) Owned by the authors, published by EDP Sciences, 2016

\title{
Energy Correlation of Prompt Fission Neutrons
}

\author{
Zs. Elter ${ }^{1, a}$ and I. Pázsit ${ }^{1, b}$ \\ ${ }^{1}$ Chalmers University of Technology, Division of Nuclear Engineering, Göteborg, Sweden, SE-41296
}

\begin{abstract}
In all cases where neutron fluctuations in a branching process (such as in multiplicity measurements) are treated in an energy dependent description, the energy correlations of the branching itself (energy correlations of the fission neutrons) need to be known. To date, these are not known from experiments. Such correlations can be theoretically and numerically derived by modelling the details of the fission process. It was suggested earlier that the fact that the prompt neutrons are emitted from the moving fission targets, will influence their energy and angular distributions in the lab system, which possibly induces correlations. In this paper the influence of the neutron emission process from the moving targets on the energy correlations is investigated analytically and via numerical simulations. It is shown that the correlations are generated by the random energy and direction distributions of the fission fragments. Analytical formulas are derived for the two-point energy distributions, and quantitative results are obtained by Monte-Carlo simulations. The results lend insight into the character of the two-point distributions, and give quantitative estimates of the energy correlations, which are generally small.

Key Words: fission neutrons; energy correlations; angular correlations.
\end{abstract}

\section{Introduction}

The statistical properties of the detection counts of neutrons, generated in subcritical multiplying media by an external source, have long been used in reactor physics and nuclear safeguards to determine parameters of the system under investigation. In reactor cores, the subcritical reactivity can be extracted from the variance to mean or from the temporal correlations of the detections; and in safeguards, the fissile mass can be determined from coincidence an multiplicity measurements [1]. The possibility of extracting such parameters is due to the fact that the branching, i.e. the neutron multiplication, generates temporal correlations between the neutrons born in the same chain.

As long as only the number of the detector counts is used, as has been the case until very recently, it is sufficient to know the number distribution of the neutrons born in a (spontaneous or induced) fission event as input data to the corresponding master equation. More precisely, since only the first few statistical moments of the detector counts are used, it is sufficient to know the moments of the fission number distribution to the same order. However, as was pointed out in [2], if the detection statistics is extended to include energy dependence, then it is not sufficient to know the number distribution and the average energy spectrum ("one-point energy distribution") of the fission neutrons. Rather, to calculate the statistics of two or three neutrons detected in coincidence in an energy dependent

\footnotetext{
ae-mail: zsolt@nephy.chalmers.se

be-mail: imre@chalmers.se
} 
case, one needs to know the two- and three-point energy distribution of the source (fission) neutrons, respectively, and so on.

Unfortunately, there are no experimental data available on the energy and angular correlations of fission neutrons. Instead, possible sources of neutron correlations are being sought in the modeling and simulation of the fission process. One possible mechanism for such correlations for spallation and fission neutrons was suggested in [3] already in 2000. It was argued that if the energies of the fission neutrons are independent of each other in any individual fission event, but the (independent and identical) energy distributions of the neutrons depend on the actual number of the neutrons (i.e the energy spectra are different for different neutron multiplicities on fission), then neutron energies become correlated when considering all possible fission events with different neutron numbers.

Recently it has also been recognised by the nuclear safeguards community that another source of the correlations between the angles and energies of the neutrons, as measured in the laboratory system, can arise from the neutron emission process. Namely, from the fact that most of the neutrons (except the so-called "pre-scission" neutrons) are emitted from the moving fission fragments as evaporation neutrons. Although the energies and emission angles of the evaporation neutrons are independent from each other in the center of mass system, their energies and directions in the lab system will be jointly affected by the fact that they are emitted from the same fission fragment, or from the two separate fission fragments, whose energies and directions are strongly correlated to each other by conservation of energy and momentum. These are now also being incorporated into numerical simulations of the intra-nuclear cascade codes $[4,5]$.

Although a large bulk of investigations were made in the safeguards community on how the emission from a moving target biases the angular directions of the neutrons in the laboratory system, the two-point (in angle) covariance function of these neutrons was neither calculated, nor measured. In addition, the effect of the emission from the moving fission fragments on the energy correlations in the lab system has not received attention yet. The purpose of this paper is to explore the properties of the energy and angular correlations of fission neutrons in the lab system through calculating the covariances, and even the two-point distributions of the neutron energies first qualitatively through analytical considerations, and then quantitatively through numerical simulations.

\section{Analytic demonstration}

In the analytical considerations, it is shown that both the energy and the angular correlations are generated in the lab system through the random properties of the fission fragment parameters.

The simplification will be used here that instead of calculating energy correlations, correlations and covariances in the velocities will be treated throughout in this section. In addition a onedimensional model will be employed, where all velocity vectors are aligned along a line, hence they can be treated as (signed) scalars. The reason for this is that the scalar velocities of the fission fragment and the emitted neutrons become additive, leading to simple convolution-type expressions for the distribution of the neutron velocities in the lab system. The same is not true for the energies, where more complicated relationships exist between the energies in the CM and the lab system.

For the sake of simplicity, only the case of two neutrons emitted from the same moving fission fragment and that the two emitted neutrons move towards the positive $x$-axis will be considered. Denote the two neutron velocities in the CM system as $v_{1}$ and $v_{2}$, and assume that they are independent and identically distributed with a probability density $f_{v}(v)$. The independence implies that one has

$$
f_{2}\left(v_{1}, v_{2}\right)=f_{v}\left(v_{1}\right) f_{v}\left(v_{2}\right) \text { and }\left\langle v_{1} v_{2}\right\rangle=\left\langle v_{1}\right\rangle\left\langle v_{2}\right\rangle
$$

The velocity of the fission fragment in the lab system is denoted by $u$, with a probability distribution $f_{u}(u)$. Here the terminology of Papoulis [6] is adopted with $f_{u}$ and $f_{v}$ denoting two different 
functions. It is also assumed that the velocity of the fission fragments is independent from the velocities of the neutrons, i.e.

$$
f_{2}(v, u)=f_{v}(v) f_{u}(u) \quad \text { and } \quad\left\langle v_{i} u\right\rangle=\left\langle v_{i}\right\rangle\langle u\rangle ; \quad \text { for } \quad i=1,2
$$

The velocities of the neutrons in the lab system will be denoted as $V_{i}, i=1,2$. In the present simple model one will have

$$
V_{i}=v_{i}+u, \quad i=1,2
$$

There are of course further assumptions behind (3), such as that the emission of the first neutron does not alter the velocity of the fission fragment. This is equivalent to neglecting the neutron mass in comparison to the mass of the fission fragments. But for the qualitative considerations these do not play a role.

The one-point and two-point distributions of the $V_{i}$ are denoted as $p_{V}(V)$ and $p_{2}\left(V_{1}, V_{2}\right)$. The task of the analytical treatment is to calculate the distributions $p_{V}(V)$ and $p_{2}\left(V_{1}, V_{2}\right)$ from the distributions $f_{v}(v)$ and $f_{u}(u)$ and the functional relationship (3) between the $v_{i}, u$ and the $V_{i}$.

Let us calculate first the covariance

$$
\sigma_{V_{1} V_{2}}^{2}=\left\langle V_{1} V_{2}\right\rangle-\left\langle V_{1}\right\rangle\left\langle V_{2}\right\rangle=\left\langle\left(v_{1}+u\right)\left(v_{2}+u\right)\right\rangle-\left\langle v_{1}+u\right\rangle\left\langle v_{2}+u\right\rangle=\sigma_{u}^{2}
$$

in terms of the variances of the neutron and the fission fragments, since it does not require the knowledge of the full probability distributions. Eq. (4) shows that the non-zero covariance between the neutron velocities is brought about by the random character, i.e. the non-zero variance of the fission fragment velocity.

It is more challenging to calculate the two-point distribution $p_{2}\left(V_{1}, V_{2}\right)$, or rather the velocity covariance function in the lab system, i.e.

$$
\operatorname{Cov}\left(V_{1}, V_{2}\right) \equiv p_{2}\left(V_{1}, V_{2}\right)-p_{V}\left(V_{1}\right) p_{V}\left(V_{2}\right)
$$

which gives a direct measure of the correlations between the neutron velocities in the lab system.

In the simple 1-D model the covariance function (5) can be easily given in analytical form. Due to the independence of the fission fragment velocity in the lab system and the neutron velocities in the CM system, the one-point and two-point densities of the neutron velocities in the lab system are given by the following expressions:

$$
p_{V}\left(V_{i}\right)=\int_{0}^{V_{i}} f_{v}\left(V_{i}-u\right) f_{u}(u) d u \quad i=1,2
$$

and

$$
p_{2}\left(V_{1}, V_{2}\right)=\int_{0}^{\max \left(V_{1}, V_{2}\right)} f_{v}\left(V_{1}-u\right) f_{v}\left(V_{2}-u\right) f_{u}(u) d u
$$

From here one obtains the result

$$
\begin{gathered}
\operatorname{Cov}\left(V_{1}, V_{2}\right)= \\
\int_{0}^{\max \left(V_{1}, V_{2}\right)} f_{v}\left(V_{1}-u\right) f_{v}\left(V_{2}-u\right) f_{u}(u) d u-\int_{0}^{V_{1}} f_{v}\left(V_{1}-u\right) f_{u}(u) d u \int_{0}^{V_{2}} f_{v}\left(V_{2}-u\right) f_{u}(u) d u
\end{gathered}
$$

It is straightforward to generalise expression (8) for the case of energy or angular correlations in a 3-D case by assuming an isotropic distribution of emitted neutrons in the CM system and even for the case when a random number of neutrons is emitted per fission. The corresponding formulae become though rapidly exceedingly involved since one has to handle the distributions of complicated nonlinear functions of random variables with known distributions (velocities and directions of the fission fragments and the emitted neutrons in the CM system). Quantitative results were therefore obtained by numerical methods. 


\section{Numerical simulations}

As indicated above, it is much more expedient to calculate the covariances and the two-point distributions by Monte-Carlo based numerical simulations in case of 3-D events and realistic fission fragment energy distributions. The details of such a simulation implemented in MATLAB is described below.

The statistical properties of the input data of the model follow largely from the choice of the fissile material. This was chosen to be uranium 235. This choice specifies, among others, the number distribution of the fission neutrons. This data is though not used in the present simulations, since always two neutrons are followed up after the fission process. One needs to specify also the energy spectrum (probability density of the neutron energies), and the energy distribution of the fission fragments.

The total kinetic energy is distributed deterministically between the two fragments according to conservation of the energy and the momentum. However, in an ensemble of many fission events, the masses of the fragments are also random variables, which adds the randomness of the fission fragment energies.

Hence the simulation starts with sampling the energies of the fission fragments. Since the kinematics of the transformation from the CM system to the lab system is given in terms of velocities, the energies are converted to speeds, then with the sampling of random angles, they are converted into velocity vectors. After the transition from $\mathrm{CM}$ to lab system is performed, the speeds are converted to energies again.

The speed of the fission fragments in the laboratory frame was thus calculated in four steps:

1. The fission yield table of the $\mathrm{U} 235$ was sampled to acquire a random fission fragment mass $m_{f f}$ (for this the JEFF 3.1 data base was used).

2. The value of the total kinetic energy $T K E$ of both fragments is considered as a normal random variable with a mean $164.4 \mathrm{MeV}$, and standard deviation $4.5 \mathrm{MeV}$.

3. The kinetic energy of the investigated fragment (the one sampled in the first step) is calculated as $K E_{f f}=\frac{235-m_{f f}}{235} T K E$.

4. Finally the random fission fragment speed is $u=\sqrt{\frac{2 K E_{f f}}{m_{f f}}}$

Hence, ultimately the probability density function of the fission fragment kinetic energy is a function of the probability distributions of the mass and the total kinetic energy.

The energies of the neutrons in the CM frame are sampled from the Maxwell spectrum. The neutron energies are independent from the energies of the fission fragments. As it was mentioned previously, the emission of the neutrons is not supposed to change the energy of the fission fragment. Such a "recoil" effect could actually be modeled in the simulations, but was not considered to be important in the present investigations, due to its very minor quantitative effect.

Having obtained the scalar velocities of the fission fragments in the lab system and the neutrons in the CM system, the neutron velocities are obtained in the lab system as

$$
V_{i}=\sqrt{v_{i}^{2}+u^{2}+2 u v_{i} \cos \left(\alpha_{i}\right) \sin \left(\beta_{i}\right)}
$$

where $\alpha_{i}$ and $\beta_{i}$ are the azimuth and polar angles respectively between the directions of the fission fragment velocity in the lab system and that of neutron $i$ in the CM system. The angles are supposed to be uniformly distributed. 


\subsection{Results}

During the simulations, not only the covariance defined in Eq. (4) was determined, but also the statistical correlation was calculated to gain a normalised estimator. The statistical correlation is defined for two random variables as the ratio of the covariance and the standard deviations:

$$
\operatorname{cor}(X, Y)=\frac{\sigma_{X, Y}^{2}}{\sigma_{X} \sigma_{Y}} \cdot 100[\%]
$$

The definition Eq. (10) gives a scalar quantity between -100 and 100 .

The simulations were done for 1-D, 2-D and 3-D events. The 1D+ case is related to a $1 \mathrm{D}$ simulation when the neutrons are emitted only towards the positive axis. In the $2 \mathrm{D}$ case, the neutron and the fission fragment velocities are not only scalar values, but vectors. The neutron velocity in the lab system is the vectorial sum of the fission fragment speed in the lab and the neutron velocity in the center-of-mass system. The 3D simulation was the generalization of the $2 \mathrm{D}$ case.

The results for the statistical correlation for the 1D, 2D and 3D cases are summarized in Table 1. Though the correlation was found positive for both cases, it is seen that the correlation is increasing with the complexity of the geometry.

Table 1: Statistical correlation for 1D, 2D and 3D cases.

\begin{tabular}{lcc}
\hline Case & Statistical correlation [\%] & $\sigma$ \\
\hline \hline 1D+ & 8.83 & 0.029 \\
1D & 0.16 & 0.031 \\
2D & 1.11 & 0.033 \\
3D & 2.23 & 0.033 \\
\hline
\end{tabular}

Fig. 1a shows the bivariate density function $p_{2}\left(E_{1}, E_{2}\right)$ of the two neutron energies in the different systems, whereas Fig. 1b displays the energy covariance map

$$
\operatorname{Cov}\left(E_{1}, E_{2}\right)=p_{2}\left(E_{1}, E_{2}\right)-p_{E}\left(E_{1}\right) p_{E}\left(E_{2}\right) .
$$

Fig. $1 \mathrm{~b}$ shows the structure of the energy correlations. As expected, these are symmetric around the $E_{1}=E_{2}$ line. There is a peak of positive correlations at low energies around $E_{1}=E_{2}$, as well as two ridges of negative (exclusive) correlations for low and constant $E_{2}$ along the $E_{1}$ a axis and a mirror image for low $E_{1}$ along the $E_{2}$ axis. These negative correlations mean that the joint occurrence of the energy pairs described above and shown by the elongated negative ridges is rather unlikely.

\section{Conclusions}

It was shown that both energy and angular correlations between fission neutrons in the lab system, emitted from the moving fission fragments, are generated due to the random energy and angular distributions of the fission fragments. Preliminary, simplified simulations show that the energy correlations generated this way are small. Further simulations in more realistic models for both energy and angular correlations will be performed in the continuation. As a first step, neutron emissions from both fission fragments can be taken into account. Furthermore, a random number of neutrons for all multiplicities can be accounted for, with also taking into account the fact that the neutron energy distributions depend on the number of neutrons emitted. 


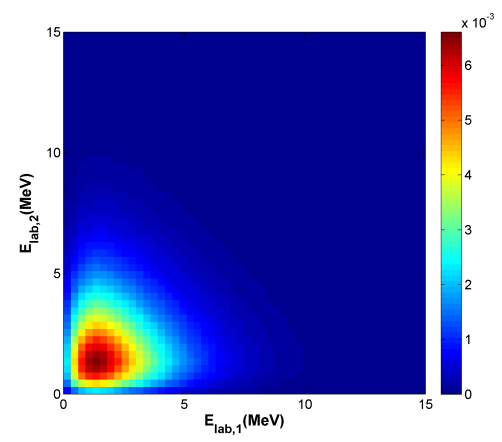

(a) Joint energy distribution

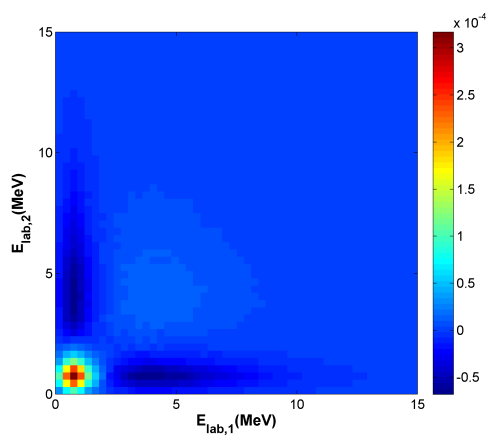

(b) Covariance map

Figure 1: Joint distributions for the realistic 3D case.

\section{Acknowledgements}

This work was financially supported by the Swedish Research Council, Grant No B0774801.

\section{References}

[1] I. Pázsit, L. Pál, Neutron Fluctuations: a Treatise on the Physics of Branching Processes, 1st edn. (Elsevier, New York, 2008)

[2] I. Pázsit, Physica Scripta 59, 344 (1999)

[3] I. Pázsit, N. Sjöstrand, V. Fhager, Nuclear Instruments and Methods in Physics Research Section A: Accelerators, Spectrometers, Detectors and Associated Equipment 452, 256 (2000)

[4] R. Vogt, J. Randrup, D.A. Brown, M.A. Descalle, W.E. Ormand, Physical Review C 85, 024608 (2012)

[5] C. Hagmann, J. Randrup, R. Vogt, Nuclear Science, IEEE Transactions on 60, 545 (2013)

[6] A. Papoulis, Probability, Random Variables and Stochastic Processes, 3rd edn. (McGraw-Hill, Inc, New York, 1991) 